\title{
Gestion forestière multi-usages en Afrique centrale : perceptions, mises en œuvre et évolutions
}

Guillaume LESCUYER ${ }^{1,2,3}$

Julienne Nadège Essoungou 4

${ }^{1}$ Cirad

UR B\&SEF

Campus international de Baillarguet 34398 Montpellier Cedex 5

France

${ }^{2}$ Cirad

UR B\&SEF

BP 2572, Yaoundé

Cameroun

${ }^{3}$ Cifor

Bureau régional pour l’Afrique centrale

Yaoundé

Cameroun

${ }^{4}$ Foder

BP 11417, Yaoundé

Cameroun

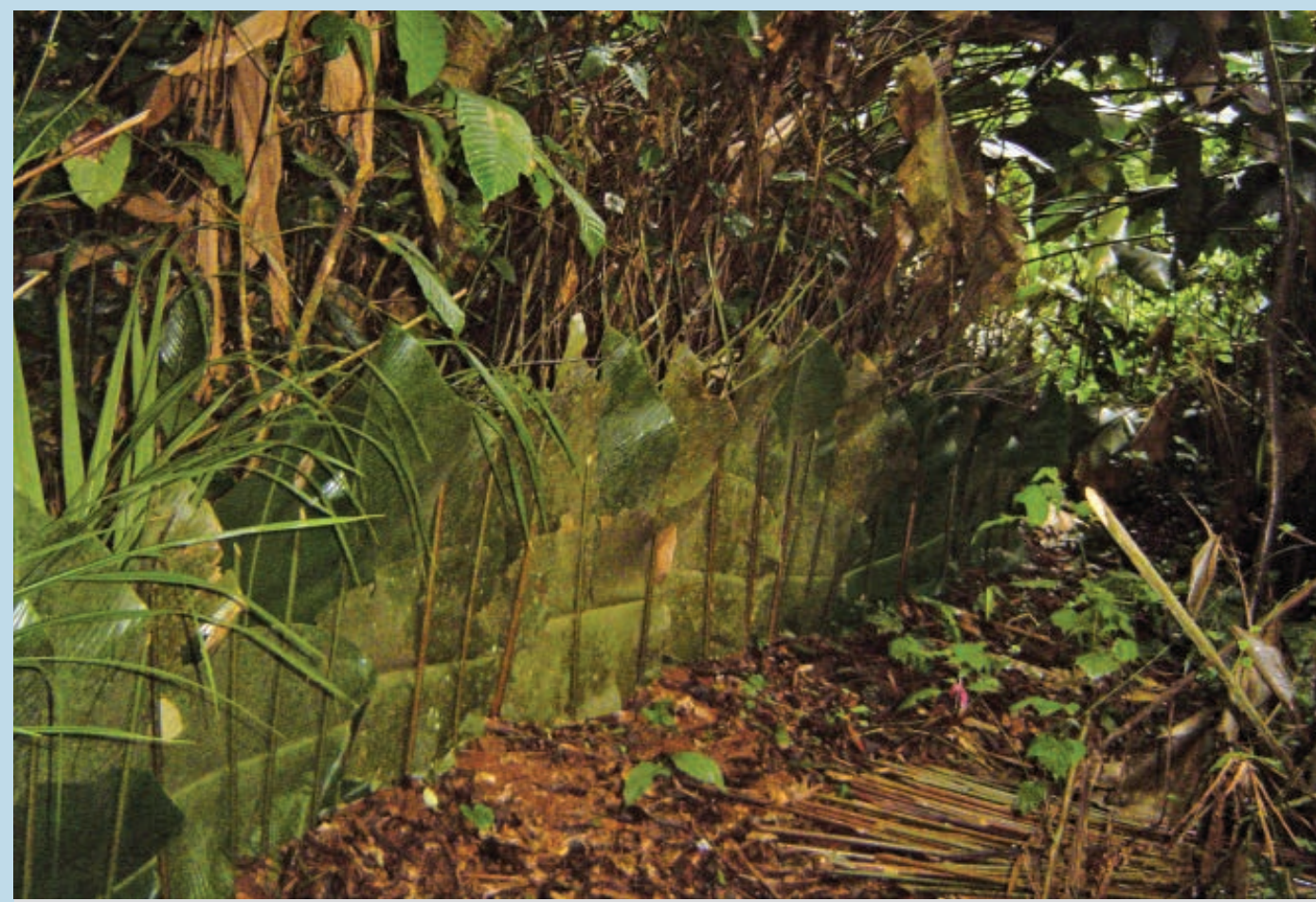

Photo 1.

Une barrière de pièges à collet dans une forêt camerounaise.

Photo G. Lescuyer. 


\section{RÉSUMÉ} GESTION FORESTIÈRE MULTI-USAGES
EN AFRIQUE CENTRALE : PERCEPTIONS,
MISES EN GUVRE ET ÉVOLUTIONS

Quoique la gestion forestière multiusages (Gfmu) soit promue par les codes forestiers d'Afrique centrale, cette approche reste mal comprise et peu mise en œuvre pour les forêts de production et les forêts communautaires. L'article présente les résultats de 62 entretiens avec des personnes impliquées dans la gestion forestière au Cameroun, au Gabon et en République démocratique du Congo, et fait ressortir trois interprétations de la Gfmu : une exploitation durable du bois intégrant secondairement les usages des autres acteurs ; une utilisation coutumière des ressources par les populations locales; une gestion planifiée et formelle de la diversité des biens et fonctions fournis par ces écosystèmes. L'analyse détaillée de huit études de cas montre en pratique que l'aménagement durable de la forêt se focalise presque toujours sur l'exploitation du bois ; quoique l'utilisation du gibier et la collecte des produits forestiers non ligneux soient systématiquement mentionnés dans les documents d'aménagement. À l'inverse, les services environnementaux - séquestration du carbone, protection des bassins versants - ou les biens publics y sont quasi absents. L'inscription de différents usages dans les documents de gestion ne suffit toutefois pas pour qu'ils soient toujours mis en œuvre sur le terrain. Trois pistes sont explorées pour renforcer l'application de la Gfmu dans le bassin du Congo : concevoir la Gfmu à l'échelle du paysage et non à celle du massif forestier ; améliorer le contenu des documents d'aménagement forestier pour y inclure et valoriser l'ensemble des usages de la forêt ; renforcer le contrôle de l'application effective des documents de gestion, grâce à la certification ou à un meilleur contrôle du respect de la légalité.

Mots-clés : gestion forestière multi-usages (Gfmu), services environnementaux, aménagement durable, Cameroun, Gabon, République démocratique du Congo.

\section{ABSTRACT}

\section{MULTIPLE USE FOREST MANAGEMENT IN CENTRAL AFRICA: PERCEPTIONS, IMPLEMENTATION AND EVOLVING PRACTICE}

Although Multiple Use Forest Management (MUFM) is promoted in Central Africa in its forest codes, the approach is not well understood and rarely implemented in either production or community forests. This article presents findings from 62 interviews with people involved in forest management in Cameroon, Gabon and the Democratic Republic of Congo, which have brought out three different interpretations of the MUFM approach: through sustainable logging integrating forest uses in other sectors as a secondary consideration, through customary uses of resources by local populations, and through formal plans for the management of forest ecosystem goods and services. A detailed analysis of eight case studies shows that in practice, sustainable forest management focuses almost exclusively on timber extraction, although hunting for game and harvesting non-timber forest products are always mentioned in management documents. On the other hand, ecosystem services carbon sequestration and catchment basin protection - and public goods are virtually never mentioned. Including different uses in management documents does not suffice to ensure that they are systematically taken into account in practice. Three possibilities are explored here for strengthening MUFM in the Congo Basin: designing MUFM approaches on the scale of the landscape rather than the forest, improving the content of management documents to include and enhance the value of all forest uses, and strengthening controls over the effective application of management documents through certification or better law enforcement.

Keywords: Multiple Use Forest Management (MUFM), ecosystem services, sustainable management, Cameroon, Gabon, Democratic Republic of Congo.

\section{RESUMEN}

\section{ORDENACIÓN FORESTAL CON FINES MÚLTIPLES EN ÁFRICA CENTRAL: PERCEPCIONES, APLICACIONES Y EVOLUCIONES}

Aunque la ordenación forestal con fines múltiples (OFFM) es fomentada por los códigos forestales de África Central, este enfoque sigue siendo mal comprendido y poco aplicado en bosques de producción y bosques comunitarios. Este artículo presenta los resultados de 62 entrevistas con personas involucradas en la ordenación forestal en Camerún, Gabón y la República Democrática del Congo en las que aparecen tres interpretaciones de la OFFFM: un aprovechamiento maderero sostenible que integra de forma secundaria los usos de otros actores; una utilización consuetudinaria de los recursos por parte de la población local; una ordenación planificada y formal de la diversidad de bienes y funciones suministrados por estos ecosistemas. El análisis detallado de ocho estudios de caso muestra, en la práctica, que el manejo sostenible del bosque se centra casi siempre en el aprovechamiento maderero; aunque la caza y la recolección de productos forestales no madereros se mencionen sistemáticamente en los documentos de manejo. Por el contrario, tanto los servicios ambientales - secuestro de carbono, protección de cuencas hidrográficas - como los bienes públicos están prácticamente ausentes. No obstante, la inclusión de los distintos usos en los documentos de ordenación no garantiza siempre su aplicación. Se exploran tres vías para reforzar la aplicación de la OFFM en la cuenca del Congo: diseñar la OFFM a escala del paisaje y no del macizo forestal; mejorar el contenido de los documentos de manejo forestal de modo que se contemplen y valoricen todos los usos del bosque; reforzar el control de la aplicación efectiva de los documentos de ordenación mediante certificación o a través de un mejor control del respeto de la normativa legal.

Palabras clave: Ordenación Forestal con Fines Múltiples (OFFM), servicios ambientales, manejo sostenible, Camerún, Gabón, República Democrática del Congo. 
Baser la gestion des forêts sur un large spectre de ressources est une recommandation ancienne en sciences forestières comme en sciences sociales (PANAYATOU, ASHTON, 1992 ; HANLEY et al., 1994). L'objectif plus récent de durabilité a renforcé l'intérêt pour une large prise en compte des biens et services ainsi que des différentes catégories d'acteurs dans la définition des modalités de gestion des ressources forestières. Cette volonté d'une gestion forestière multi-usages (Gfmu) est aujourd'hui largement dominante dans la littérature spécialisée (WANG, WILSON, 2007 ; GARCIA-FERNANDEZ et al., 2008) mais peu mise en œuvre dans la gestion pratique des forêts tropicales, d'où l'intitulé d'un numéro récent de la revue Forest Ecology and Management : «Multiple use management of tropical production forests: How can we move from concept to reality? » (GUARIGUATA et al., 2012).

Depuis une vingtaine d'années, l'ensemble des lois forestières des pays du bassin du Congo a été revu et promeut une gestion intégrée et durable des ressources forestières. Une première mesure a été la mise en place des schémas nationaux d'aménagement de l'espace forestier, qui délimitent des espaces essentiellement consacrés à l'exploitation des ressources ligneuses (dénommés généralement «forêts de production »), des aires protégées et des espaces agroforestiers (ou domaine forestier non permanent) dont la vocation est de permettre aux populations locales de répondre à leurs besoins (ASSEMBE et al., 2011). À l'échelle nationale, la plupart des usages des ressources forestières sont donc pris en compte sous la forme d'une répartition d'espaces spécialisés qui se côtoient sans se combiner. L'enjeu d'une mise en œuvre effective de la Gfmu se situe en conséquence à une échelle géographique inférieure, celle de ces massifs spécialisés.

En raison de leur aménagement formel - contrairement aux espaces agroforestiers - et de leur compatibilité avec de nombreuses formes d'usage - contrairement aux parcs nationaux - les forêts de production sont les plus susceptibles d'être gérées selon les principes de la Gfmu. Une telle Gfmu repose alors sur la rédaction puis la mise en œuvre d'un document formel d'aménagement qui explicite et régule les usages des ressources.

Sans définir précisément le concept de Gfmu, les lois forestières des pays d'Afrique centrale encouragent la valorisation des différents biens et services de la forêt dans l'aménagement des forêts de production. Elles entérinent, tout d'abord, le principe de participation des parties prenantes au processus d'aménagement des forêts (LESCUYER, 2006). Elles reconnaissent également les droits d'usage des communautés sur les ressources, tout en limitant la commercialisation des produits tirés de ces activités coutumières. Elles mentionnent enfin plusieurs biens et services qui doivent ou peuvent être considérés lors de l'aménagement de la forêt de production. Il s'agit notamment des produits forestiers non ligneux (Pfnl), de la diversité biologique animale et végétale, de l'écotourisme, des zones sacrées, de la recherche, et par- fois de l'agroforesterie. Les services environnementaux, comme la séquestration du carbone ou le maintien de la qualité du réseau hydrique, ne font pas encore l'objet d'une attention particulière dans le processus d'aménagement forestier, quoique il existe déjà certaines initiatives privées tentant de les valoriser (LESCUYER et al., 2009). Aujourd'hui, outre l'aménagement des ressources ligneuses commerciales, seules la gestion du gibier (photo 1), la valorisation des Pfnl, et l'identification des sites à haute valeur de conservation (photo 2) sont des rubriques obligatoires des plans d'aménagement forestier dans le bassin du Congo.

Cependant, l'intégration de ces usages multiples dans les documents de gestion se traduit encore rarement par la mise en œuvre d'une Gfmu sur le terrain (NASI et al., 2012). En pratique, les concessionnaires forestiers demeurent focalisés sur l'exploitation des ressources ligneuses, indépendamment des dispositions inscrites dans les plans d'aménagement concernant la valorisation des autres types d'usage.

Cet article montre la difficulté dans trois pays d'Afrique centrale à mettre en œuvre la Gfmu dans les forêts de production, en dépit d'un cadre légal favorable, à défaut d'être véritablement incitatif. Après avoir introduit le contexte géographique et présenté les méthodes d'enquête, une analyse de la compréhension de la Gfmu par les acteurs est réalisée. L'inscription de la Gfmu dans huit documents d'aménagement forestier est ensuite décrite sur la base d'études de cas dispersées au Cameroun, au Gabon et en République démocratique du Congo (Rdc). Enfin, trois mécanismes susceptibles d'accroître à moyen terme l'adoption de la Gfmu par les acteurs clés sont envisagés. 


\section{Sites d'étude et méthodes d'enquête}

Le choix du Cameroun, du Gabon et de la Rdc pour étudier la mise en œuvre de la Gfmu a été guidé par l'importance du potentiel forestier en termes de surface, d'avancement dans le processus d'aménagement forestier et de contribution aux ressources économiques du pays. Ces trois pays offrent une image représentative des modes d'exploitation forestière, qui sont relativement homogènes d'un point de vue légal, technique, écologique, social et économique en Afrique centrale (DE WASSEIGE et al., 2012). Deux types de données ont été collectés dans les trois pays :

- les données secondaires ont été obtenues à travers l'analyse documentaire du cadre législatif, institutionnel et socioéconomique ;

- les données primaires, par le biais d'entretiens individuels auprès des différents acteurs.

Deux supports d'enquête ont été utilisés. D’une part, une grille d'entretien individuel a permis d'apprécier les perceptions de la Gfmu auprès de 62 personnes de nationalités et de professions différentes (tableau I). Cependant, ces 62 personnes sont avant tout impliquées ou concernées par la gestion formelle des ressources forestières et développent des perceptions qui divergent probablement en partie de celles des utilisateurs quotidiens des ressources forestières. De même, seules 6 femmes ont été interrogées sur les 62 personnes rencontrées, ce qui peut introduire un biais en faveur d'une vision masculine de la Gfmu.

D’autre part, dans le cadre d'un entretien fermé, six gestionnaires de forêt de production et deux responsables de forêt communautaire (figure 1) ont décrit de quelle manière ils avaient intégré la Gfmu dans leurs documents de gestion des massifs forestiers. Ces entretiens ont été complétés par la consultation des documents de gestion forestière afin de trianguler l'information obtenue lors de l'entretien. Chacun de ces questionnaires durait environ 45 minutes. Ils étaient administrés soit dans les bureaux des personnes interrogées, soit à proximité des zones en exploitation.

Les données collectées lors de ces entretiens étaient essentiellement qualitatives. Leur classification s'est faite sur la base des vocables et des références contenus dans les discours des personnes interrogées.

\section{Résultats : perceptions et intégration de la Gfmu dans les documents d'aménagement forestier}

L'absence de définition claire de la Gfmu dans les codes forestiers des pays du bassin du Congo favorise la multiplicité des points de vue sur le sens et le contenu de cette approche. Trois interprétations de la Gfmu ressortent des entretiens réalisés dans les trois pays sélectionnés (tableau II).

L'interprétation de la Gfmu dépend avant tout du type d'acteur, et peu du contexte national. Sans surprise, les compagnies et les administrations forestières concentrent leur attention sur le bois d'œuvre, qui constitue la principale ressource gérée formellement en Afrique centrale, par le biais des plans d'aménagement forestier. À l'inverse, les représentants des populations locales se réfèrent généralement au savoirfaire des communautés en matière de gestion à long terme de leur patrimoine naturel et culturel et à la perturbation des systèmes socio-écologiques locaux par l'arrivée de nouveaux acteurs comme les concessionnaires. Enfin, une troisième communauté épistémique, largement dépendante de financements internationaux, met davantage l'accent sur la gestion intégrée des forêts avec un accent particulier sur la conservation de la biodiversité ou la séquestration du carbone.

Dans la mise en œuvre de la gestion forestière en Afrique centrale, telle que promue par les codes forestiers, la première interprétation de la Gfmu - exploitation du bois d'œuvre associée au maintien d'autres bénéfices secondaires - est largement dominante, comme le montrent les entretiens tenus avec huit responsables de concession forestière ou de forêt communautaire. Sur ces huit études de cas, une seule initiative de foresterie communautaire en Rdc focalise ses efforts sur la valorisation des Pfnl (photo 3 ), en prenant en compte de manière secondaire l'agriculture, la chasse et la pêche. Malgré l'intérêt économique que peut avoir l'exploitation des Pfnl pour les populations rurales (BEAUCHAMP, INGRAM, 2011), cette activité est rarement privilégiée lorsque les communautés formalisent et officialisent leur gestion locale des ressources forestières, par exemple sous la forme de forêt communautaire. Au Cameroun, les communautés choisissent majoritairement l'exploitation du bois d'œuvre comme principal usage de leur forêt communautaire (EZZINE DE BLAS et al., 2009).

\section{Tableau I. \\ Catégories d'acteurs interrogés.}

Catégories d'acteurs

Compagnie forestière

Leaders communautaires

Responsable de projet

Chercheur

Cadre de l'administration forestière

Responsables d'organisation non gouvernementale

Total

\begin{tabular}{|c|c|c|} 
Cameroun & Gabon & République démocratique du Congo \\
\hline 5 & & \\
\hline 5 & 3 & 4 \\
\hline 5 & 0 & 1 \\
\hline 3 & 7 & 2 \\
\hline 2 & 2 & 1 \\
\hline 5 & 4 & 3 \\
\hline 25 & 5 & 5 \\
\hline
\end{tabular}




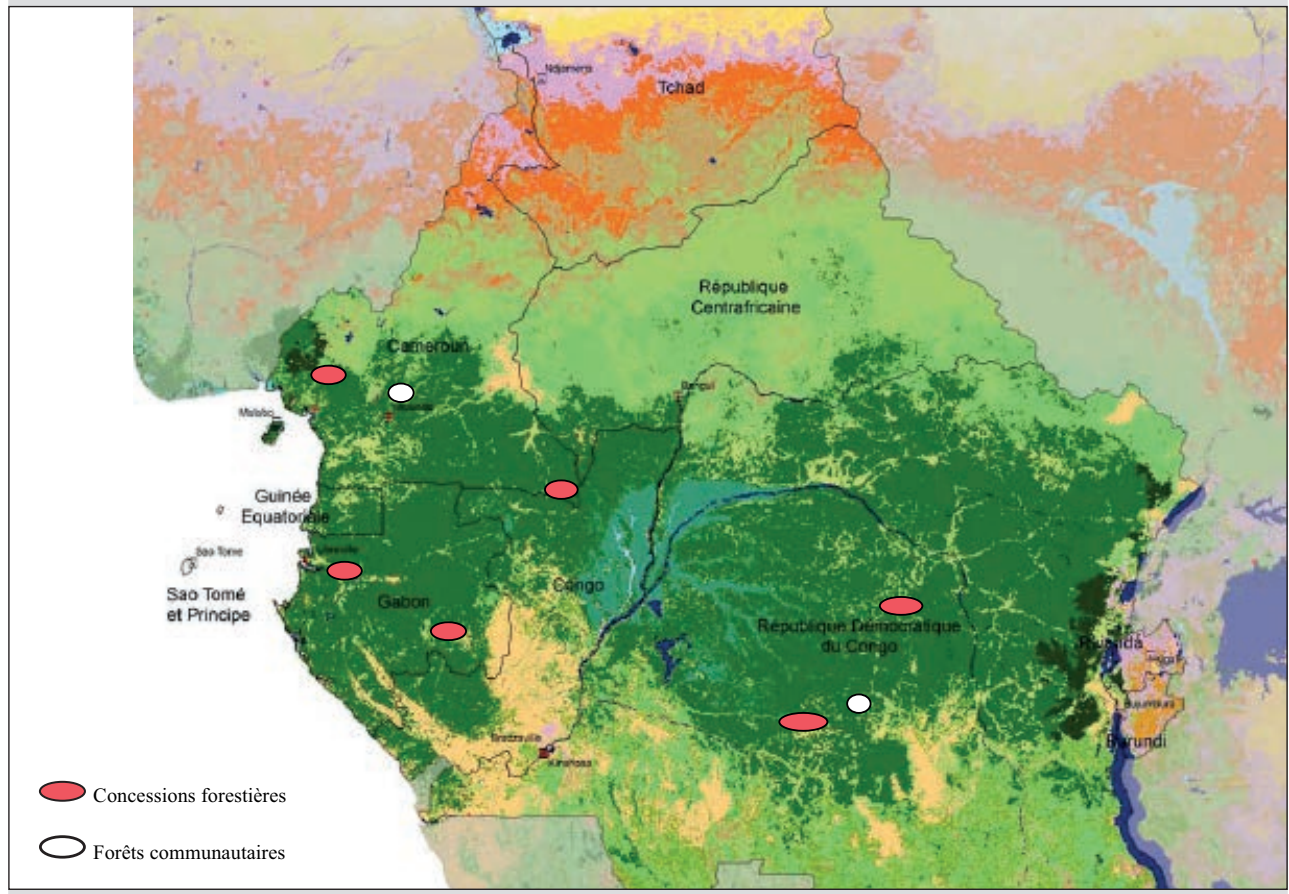

Figure 1.

Sites d'étude au Cameroun, au Gabon et en République démocratique du Congo

(fonds de carte : DE WASSEIGE et al., 2012).

Au total, outre l'exploitation du bois qui reste l'usage dominant dans les documents de gestion forestière, deux autres usages sont presque systématiquement pris en compte : l'utilisation du gibier et la cueillette. Ces ressources sont intégrées dans le processus d'aménage. ment des forêts et font l'objet d'inventaires, dont la qualité est cependant parfois discutable. À l'inverse, très peu de considération est accordée aux biens publics et aux services globaux comme la protection des bassins versants ou la séquestration du carbone - dans ces documents de gestion forestière.

L'intégration de plusieurs usages forestiers dans les documents de gestion, et l'établissement de règles spécifiques d'usage, ne signifient toutefois pas qu'elles soient automatiquement mises en œuvre dans les massifs forestiers, comme l'indiquent d'autres travaux réalisés dans le bassin du Congo (VAN-

Sur cet échantillon de huit études de cas, six concessions forestières et une forêt communautaire privilégient l'exploitation du bois d'œuvre. Plusieurs autres usages sont intégrés dans ces documents de gestion : l'exploitation et/ou la conservation du gibier (7 cas), la cueillette des Pfnl (7 cas), l'agriculture ( 5 cas), le bois de chauffe (3 cas), la recherche (2 cas), le sciage artisanal (1 cas) et la séquestration du carbone (1 cas).
DENHAUTE, DOUCET, 2006 ; (CROIZER, TRÉFON, 2007). Par exemple, LESCUYER et al. (2012) montrent pour neuf concessions forestières implantées au Cameroun que l'application des mesures encadrant les usages locaux des ressources forestières est généralement peu satisfaisante : si le contrôle des pratiques agricoles est souvent effectif parce que leur extension peut mettre en péril des ressources ligneuses, les mesures censées réguler la chasse, la cueil-

Tableau II.

Perceptions de la gestion forestière multi-usages (Gfmu).

Interprétation de la Gfmu

Exploitation du bois d'œuvre associée au maintien d'autres bénéfices secondaires

Utilisations coutumières informelles des ressources forestières

Gestion formalisée et intentionnelle de l'ensemble des ressources forestières

Argumentaire
L'exploitation industrielle du bois
d'œuvre peut être aisément combinée
avec d'autres types d'usages, tant que
ceux-ci ne nuisent pas à l'exploitation
Les populations locales ont démontré
avoir géré l'ensemble des ressources
forestières de manière durable depuis
des siècles
Optimiser l'utilisation des ressources
forestières nécessite une gestion
formelle qui explicite et organise la
combinaison ou la complémentarité
de ces usages

Acteurs portant ce discours

Sociétés forestières, administrations forestières

Représentants des communautés, organisation non gouvernementale

Gestionnaires de projet, instituts de recherche, organisation non gouvernementale 


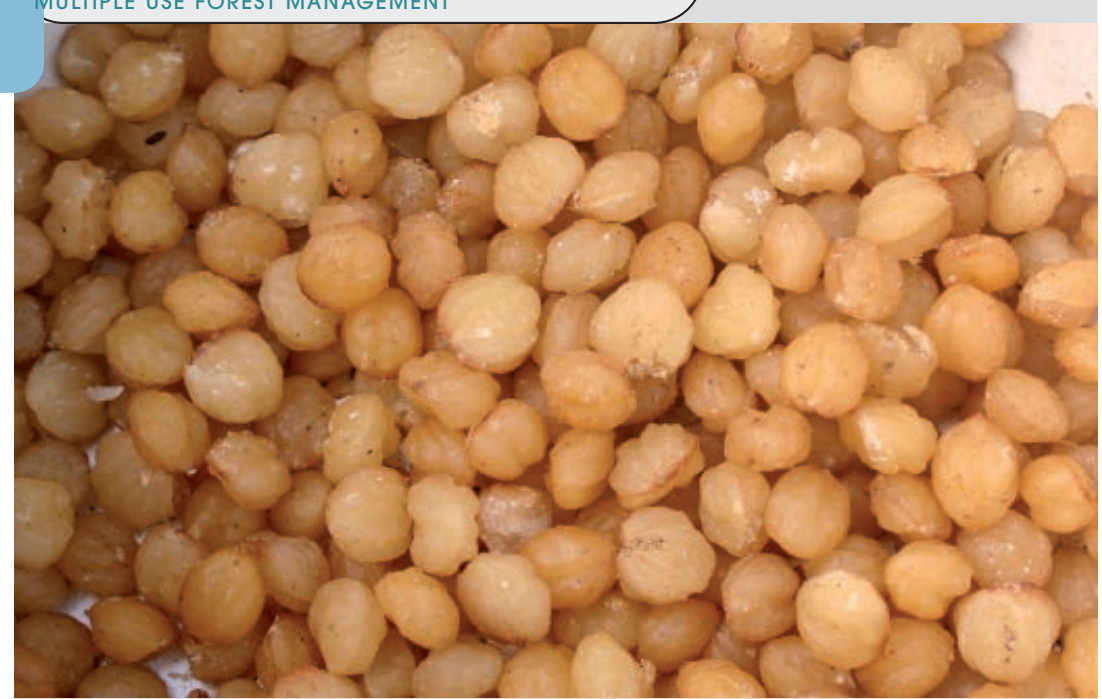

Photo 3.

Un des Pfnl exploités en Afrique centrale pour l'alimentation : le ndjansan, Ricinodendron heudelotii.

Photo G. Lescuyer.

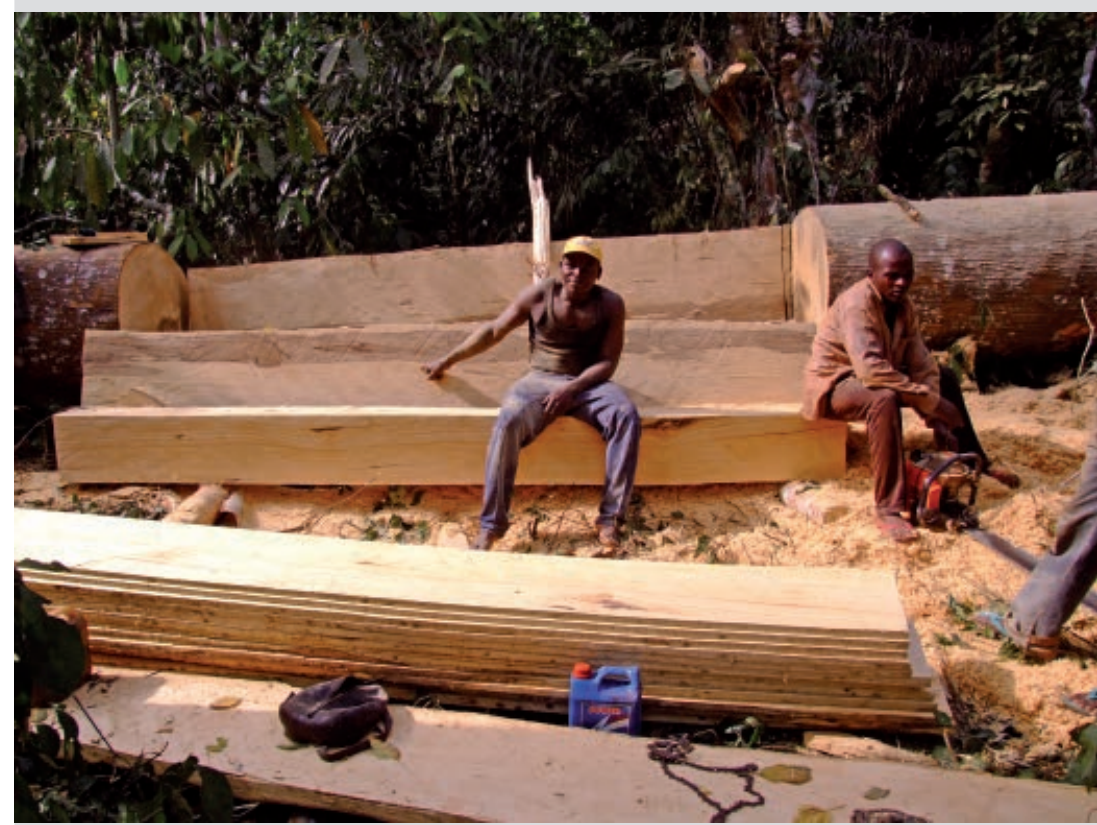

Photo 4.

Une équipe de scieurs artisanaux se reposant sur un ayous, Triplochiton scleroxylon.

Photo G. Lescuyer.

\section{Discussion : comment promouvoir la Gfmu dans le bassin du Congo ?}

Trois approches paraissent propices pour favoriser la Gfmu dans les pays du bassin du Congo : concevoir la Gfmu à l'échelle du paysage plutôt qu'au seul niveau du massif forestier, améliorer le contenu des documents de gestion forestière, et renforcer le contrôle de leur application. Ces trois propositions sont discutées ci-dessous.

En l'absence d'incitations réglementaires ou économiques à promouvoir la Gfmu dans les permis forestiers, un changement d'échelle géographique peut être envisagé afin d'optimiser les relations entre des espaces spécialisés par exemple au sein d'un "paysage », entendu comme un espace géographique défini officiellement sur la base de caractéristiques et d'objectifs spécifiques et qui présente une diversité d'usages méritant d'être mieux coordonnés (BILLAND, NASI, 2008 ; NASI et al., 2012). De tels paysages existent déjà en Afrique centrale : il s'agit par exemple des 13 paysages mis en place sous la tutelle de l'Usaid ou des forêts modèles promues par le service canadien de coopération, qui sont chacun organisés autour d'une aire protégée mais qui recouvrent aussi presque toujours des concessions forestières et des zones agroforestières (DE WASSEIGE et al., 2012). Plusieurs de ces paysages produisent des résultats intéressants en appuyant la certification de concessions forestières, en mettant en place des plates-formes de gouvernance (ENDAMANA et al., 2010 ; OSSIE OMPENE et al., 2011), ou en expérimentant des formes nouvelles de gestion de ressources, comme les Zones d'intérêt cynégétique à gestion communautaire dans le Sud-Est du Cameroun (BIGOMBE LOGO, 2010). Ces montages institutionnels reconnus par l'administration demeurent toutefois très dépendants des financements internationaux et de la présence d'opérateurs d'appui. Bénéficiant du soutien financier de la communauté internationale, certains de ces paysages constituent un environnement propice à la pro-

lette et le sciage artisanal (photo 4) sont rarement mises en œuvre par les gestionnaires des concessions. Ce constat est partagé à l'échelle de la sous-région par VAN VLIET et NASI (2008) pour la chasse et par NASI et al. (2012) pour la biodiversité au sens large. Trois principales raisons expliquent la faible application de ces dispositions des plans d'aménagement forestier : un coût trop élevé du contrôle, une réglementation qui est source de conflit avec les populations locales, un faible impact sur les espèces ligneuses commerciales. Dans les faits, en dépit des réglementations inscrites dans les plans d'aménagement forestier, les usages locaux des ressources forestières sont généralement tolérés par les concessionnaires plutôt qu'encadrés ou régulés. motion de la Gfmu. Pourtant ces schémas d'aménagement de méso-territoire peuvent disparaître aussi vite que les financements qui les ont créés, ce qui fragilise leur crédibilité et empêche les acteurs locaux de les considérer comme le cadre naturel de leurs interventions et investissements.

Comme indiqué précédemment, les lois et réglementations forestières en Afrique centrale prévoient la mise en œuvre d'une Gfmu. Le cadre juridique ne constitue globalement pas une entrave à la Gfmu, si ce n'est l'interdiction de commercialisation qui s'applique aux produits tirés de l'exercice des droits coutumiers. Toutefois, plusieurs organisations, comme la Fao, s'activent pour faire reconnaître l'exercice de droits d'usage commercial et il est probable que cette 
disposition soit acceptée dans le cadre actuel des révisions des lois forestières, au Cameroun notamment. L'exercice de ces droits d'usage commercial doit rester compatible avec le prélèvement durable de la ressource, en établissant par exemple des règles simples d'utilisation applicables soit dans le temps (saison de nonchasse), soit dans l'espace (respect des sites à haute valeur de conservation), soit aux techniques employées (pas de chasse au fusil, pas de poison, etc.), soit aux statuts des usagers (par exemple les seuls résidents au village depuis plus de dix ans). La faible présence des agents de l'État sur le terrain signifie une implication plus forte des acteurs locaux dans la mise en œuvre et le contrôle de ces règles, qu'il s'agisse des opérateurs privés, des populations résidentes et de la société civile.

Dans le contexte légal actuel, les documents de gestion forestière sont probablement les meilleurs supports pour parvenir à accorder les interprétations divergentes de la Gfmu et ce pour trois raisons : ils sont normalement le produit d'une concertation entre les acteurs concernés par l'aménagement ; ils peuvent intégrer de nombreux usages envisagés dans les réglementations forestières ; ils constituent un engagement formel des exploitants et des administrations. Améliorer le contenu de ces documents de gestion apparaît comme la solution la plus acceptable politiquement et la moins coûteuse à court et moyen terme pour favoriser de manière concrète l'adoption de la Gfmu. Une telle évolution requiert d'amender le cursus de formation des gestionnaires - privés et publics - encore trop ancré dans les sciences forestières. Par contre, cette stratégie n'implique pas de revoir le système actuel de tenure foncière - où l'État est propriétaire des terres - qui est généralement considéré comme un frein important à l'implication des populations locales dans la gestion forestière (RIST et al., 2012), mais qui constitue aussi un facteur d'unité nationale aux yeux des administrations et des élus. Plutôt qu'une modification institutionnellement compliquée de la tenure foncière, l'amélioration du contenu des documents de gestion forestière pourrait confirmer de nombreux droits d'usage sur les ressources - et non sur le sol - et sécuriser leur accès et leur utilisation à long terme par les populations locales (photo 5).

Enfin, la révision régulière de ces documents de gestion permet d'intégrer rapidement de nouveaux usages, comme la séquestration du carbone, dans l'utilisation des massifs forestiers. L'avantage d'introduire ces services environnementaux dans les documents de gestion forestière peut aussi être d'ordre financier. Cela devrait permettre à plus ou moins long terme aux gestionnaires de bénéficier de financements privés liés à la lutte contre le changement climatique (NASI et al., 2012). Il pourrait être aussi envisagé que les États mettent en place des mesures incitatives à la production de ces biens et services publics, par exemple sous la forme de dégrèvements fiscaux.

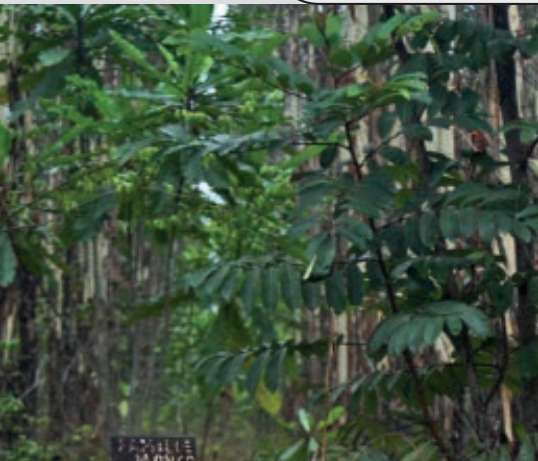

ATTENTION I!!! DOMAINE PRIVE DELA TILL TCHINGANGA MATCHNOL $\begin{aligned} & 546-49-33 \\ & 550-55-87\end{aligned} 669-73-40$
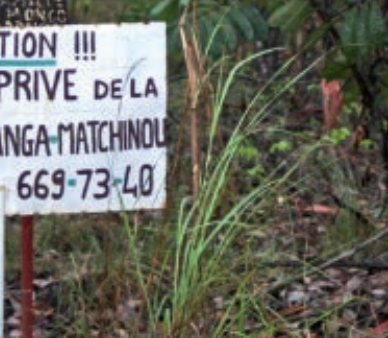

Photo 5.

Panneau indiquant la possession d'une parcelle forestière à proximité de Pointe-Noire.

Photo G. Lescuyer.

L'amélioration des documents de gestion forestière est de peu d'utilité s'ils ne sont pas appliqués sur le terrain, ce qui suppose qu'ils soient rigoureusement et régulièrement contrôlés. Ce n'est globalement pas le cas aujourd'hui en Afrique centrale, sauf pour l'exploitation du bois d'œuvre dans les grandes concessions forestières. L'extension de la certification forestière et la mise en œuvre des Accords de partenariat volontaire (Apv) ${ }^{1}$ dans les pays d'Afrique centrale (Cameroun, Congo, République centrafricaine) contribuent à améliorer la mise en œuvre réelle de ces documents de gestion.

La certification forestière, notamment celle du Forest Stewardship Council (Fsc), est souvent reconnue comme l'un des éléments qui favorisent le développement de la Gfmu (GARCIA-FERNANDEZ et al., 2008 ; NASI et al., 2012) puisque plusieurs de ses principes obligent à prendre en compte les différentes fonctions de la forêt, même si l'interprétation de ces critères par les bureaux certificateurs laisse une certaine marge quant à leur mise en œuvre (CERUTTI et al., 2011 ; RIST et al., 2012). L'avantage majeur de la certification forestière réside en réalité dans l'effectivité et la régularité des contrôles d'application - et dans l'assurance de sanctions en cas de manquement - des mesures prises dans les documents de gestion, notamment en matière de promotion ou de respect des usages multiples des ressources forestières.

La mise en œuvre des Apv devrait, à terme, favoriser la même tendance en garantissant la légalité de tous les produits forestiers et en développant un système de traçabilité de ces produits. Cela implique un contrôle régulier et sérieux de la légalité des opérations conduites dans les permis forestiers et, en conséquence, une réelle mise en œuvre des dispositions des documents de gestion en matière d'usages multiples des ressources forestières.

1 Les Accords de partenariat volontaire sont des accords bilatéraux entre l'Union européenne et les pays exportateurs de bois tropical. Leur but est d'améliorer la gouvernance des forêts et de garantir que le bois importé par l'UE provient de sources légales telles que définies dans les pays producteurs. 


\section{Conclusion}

Pour certaines catégories d'acteurs, la gestion forestière multi-usages reste encore aujourd'hui une conception intellectuellement intéressante d'utilisation durable de la forêt mais peu opérationnelle en raison du contexte économique, technique et administratif en Afrique centrale. De ce point de vue, le bois d'œuvre demeure la seule ressource forestière disposant de débouchés marchands importants, dont l'exploitation est fondée sur un corpus technique fiable, et qui fournit une contribution significative aux économies nationales. Ainsi, malgré l'ouverture théorique du processus d'aménagement forestier à d'autres ressources forestières, c'est pourtant presque toujours l'exploitation du bois d'œuvre qui, en pratique, retient l'attention des gestionnaires de concessions forestières comme de forêts communautaires (photo 6). Cette application du modèle de l'aménagement forestier durable est toutefois battue en brèche depuis quelques années en Afrique centrale avec l'arrivée de nouveaux investisseurs intéressés par des usages alternatifs des espaces forestiers : de nombreux massifs forestiers font aujourd'hui face aux pressions venant de projets agro-industriels, de projets miniers ou d'initiatives de non-émission de gaz à effet de serre, dont les bénéfices financiers peuvent être beaucoup plus élevés que les revenus tirés de l'exploitation du bois. Dans ce contexte nouveau de compétition sur les espaces forestiers, la Gfmu peut permettre d'accroître les bénéfices économiques tirés de l'utilisation durable de la forêt tout en renforçant la légitimité sociale et politique de ce mode d'aménagement du territoire, en associant les acteurs locaux. Plusieurs initiatives, comme le développement de la certification Fsc ou la mise en œuvre prochaine des Apv permettent actuellement d'appuyer la mise en œuvre de la Gfmu, souvent avec le soutien de financements internationaux. Ces opportunités sont à saisir à court terme pour mieux faire face à la concurrence d'autres modes d'usage de ces espaces.

\section{Remerciements}

Cette recherche a été conduite grâce à un financement octroyé par la Fao et l'Oibt. Les auteurs souhaitent également remercier deux relecteurs anonymes dont les contributions ont permis d'améliorer le contenu de cet article.
ASSEMBE MVONDO S., EBA'A ATYI R., LESCUYER G., WARDELL A., 2011. Sustainable forest management based on State practice in Central Africa countries. Nature \& Faune, 26 (1): 45-48.

BEAUCHAMP E., INGRAM V., 2011. Impacts of community forests on livelihoods in Cameroon: Lessons from two case studies. International Forestry Review, 13 (3): 1-13.

BIGOMBE LOGO P., 2010. Governance of decentralized forest revenue in Central Africa: For better or for Worse. In: German L., Karsenty A., Tiani A. M. (éds). Governing Africa's forests in a globalized World. Londres, Royaume-Uni, Earthscan, 174-189.

BILLAND A., NASI R., 2008. Production dans les forêts de conservation, conservation dans les forêts de production : vers des forêts tropicales durables, à partir du cas de l'Afrique centrale. In : Meral P., Castellanet C., Lapeyre R. (éds). La gestion concertée des ressources naturelles L'épreuve du temps. Paris, France, Gret/Karthala, 201-219.

CERUTTI P. O., TACCONI L., NASI R., LESCUYER G., 2011. Legal versus certified timber: Preliminary impacts of forest certification in Cameroon. Forest Policy and Economics, 13: 184-190. CROIZER C., TRÉFON T., 2007. Quel avenir pour les forêts de la République démocratique du Congo ? Instruments et mécanismes innovants pour une gestion durable des forêts. Bruxelles, Belgique, Ctb, Reflection and Discussion Paper 2007/01, 83 p.

DE WASSEIGE C., DE MARCKEN P., BAYOL N., HIOL HIOL F., MAYAUX P., DESCLÉE B., NASI R., BILLAND A., DEFOURNY P., EBA'A ATYI R., 2012. Les forêts du bassin du Congo - État des Forêts 2010. Luxembourg, Office des publications de l'Union européenne, 276 p.

ENDAMANA D., BOEDHIHARTONAO A. K., BOKOTO B., DEFO L., EYEBE A., NDIKUMAGENGE C., NZOOH Z., RUIZ-PÉREZ M., SAYER J., 2010. A framework for assessing conservation and development in a Congo Basin Forest Landscape. Tropical Conservation Science, 3 (3): 262-81.

EZZINE DE BLAS D., RUIZ PÉREZ M., SAYER J. A., LESCUYER G., NASI R., KARSENTY A., 2009. External influences on and conditions for community logging management in Cameroon. World Development, 37 (2): 445-56.

GARCIA-FERNANDEZ C., RUIZ PEREZ M., WUNDER S., 2008. Is multiple-use forest management widely implementable in the tropics? Forest Ecology and Management, 256: 1468-1476.

GUARIGUATA M. R., SIST P., NASI R., 2012. Multiple use management of tropical production forests: How can we move from concept to reality? Forest Ecology and Management, 263: 170-174.

HANLEY N., SHOGREN J. F., WHITE B., 1994. Environmental Economics in Theory and Practice. Londres, Royaume-Uni, MacMillan, $356 \mathrm{p}$. 


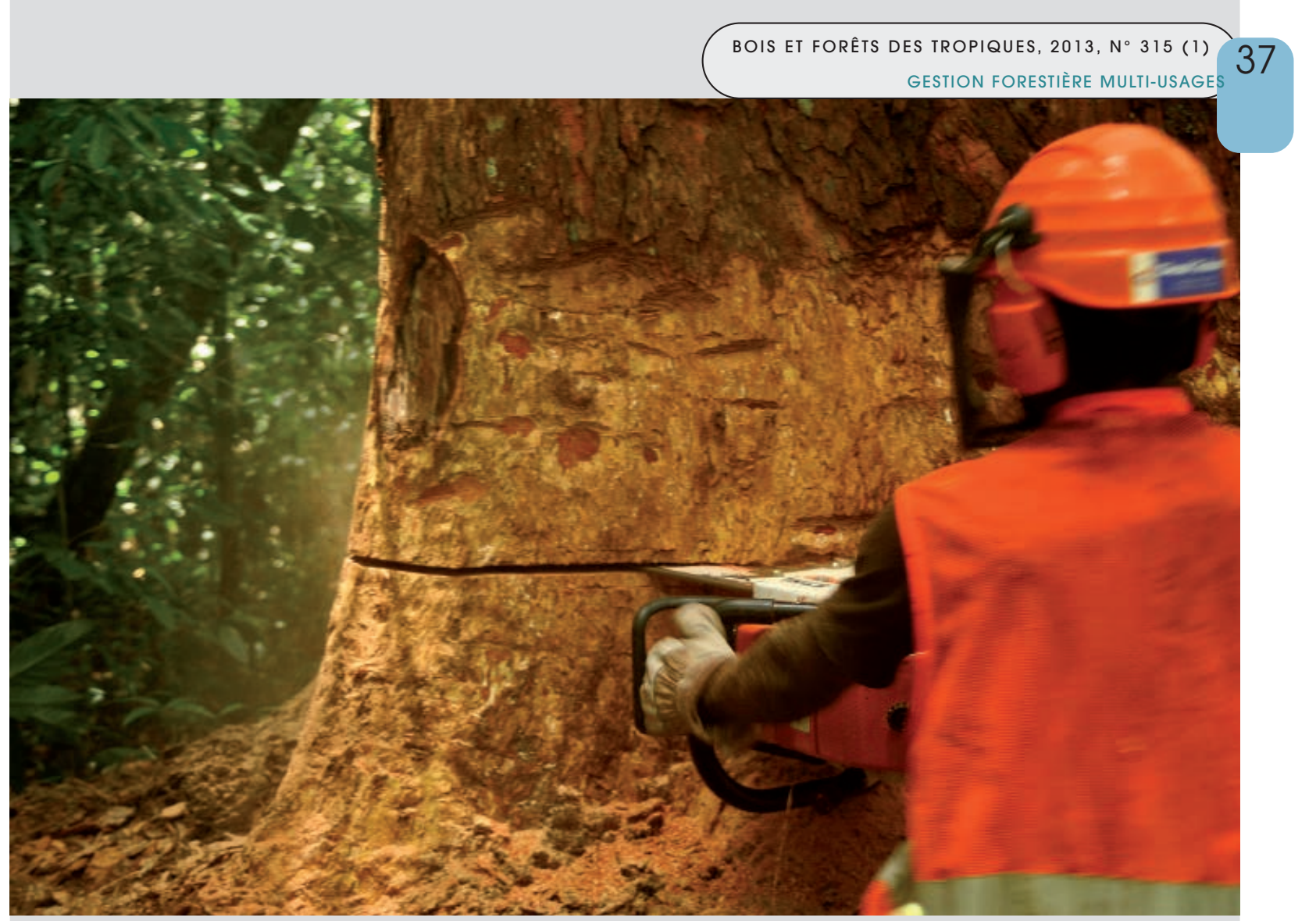

Photo 6.

Abattage d'un azobé, Lophira alata, dans une forêt gabonaise.

Photo G. Lescuyer.

LESCUYER G., 2006. Formes d'action collective pour la gestion locale de la forêt camerounaise : organisations «modernes » ou institutions « traditionnelles »? Vertig0, 6 (3) : http://vertigo.revues.org/index8029.html.

LESCUYER G., KARSENTY A., EBA'A ATYI R., 2009. Un nouvel outil de gestion durable des forêts d'Afrique centrale : les paiements pour services environnementaux. In : De Wasseige C., Devers D., De Marcken P., Eba'a Atyi R., Nasi R., Mayaux P. (éds). Les Forêts du Bassin du Congo - État des Forêts 2008. Luxembourg, Office des publications de l'Union européenne, 131-144.

LESCUYER G., ASSEMBE MVONDO S., ESSOUNGOU J. N., TOISON V., TRÉBUCHON J.-F., FAUVET N., 2012. Logging concessions and local livelihoods in Cameroon: from indifference to alliance? Ecology and Society, 17 (1): art. 7.

NASI R., BILLAND A., VAN VLIET N., 2012. Managing for timber and biodiversity in the Congo Basin. Forest Ecology and Management, 268: 103-111.

OSSIE OMPENE G., PA'AH P. A., BOUKI T., NJOMKAP J. C., GAGOE TCHOKO J., DIAW M. C., 2011. Multi-actor Partnership and Sustainability Management of Biodiversity: The Case of the World Heritage of the Dja Faunal Reserve. In: Adapting to change: the state of conservation of World Heritage forests in 2011. Paris, France, Unesco, World Heritage Center, World Heritage Series n 30, 86-90.
PANAYATOU T., ASHTON P., 1992. Not by timber alone: Economics and ecology for sustaining tropical forests. Washington D.C., États-Unis, Island Press, 280 p.

RIST L., SHANLEY P., SUNDERLAND T., SHEIL D., NDOYE O., LISWANTI N., TIEGUHONG J., 2012. The impacts of selective logging on non-timber forest products of livelihood importance. Forest Ecology and Management, 268: 57-69.

VANDENHAUTE M., DOUCET J.-L., 2006. Étude comparative de vingt plans d'aménagement approuvés au Cameroun. Yaoundé, Cameroun, German Technical Cooperation, 57 p.

VAN VLIET N., NASI R., 2008. Mammal distribution in a Central African logging concession area. Biodiversity Conservation, 17 (5): 1241-1249.

WANG S., WILSON B., 2007. Pluralism in the economics of sustainable forest management. Forest Policy and Economics, 9: 743-750. 